\title{
EM-Based Optimal Maximal Ratio Diversity Combiner for Constant Envelope Signals
}

\author{
Ahmed El-Sayed El-Mahdy*
}

Electrical Engineering Department, MTC, Cairo, Egypt

\begin{abstract}
An optimal maximal ratio combiner (MRC) based on the expectation-maximization (EM) algorithm is developed for noisy constant envelope signals transmitted over a Rayleigh fading channel. Instead of using a transmitted pilot signal with the data to estimate the combiner gains, the EM algorithm is used to perform this estimation. In the developed MRC, estimation of the transmitted data sequence is performed also by the EM algorithm. Estimation using the EM algorithm provides an iterative solution to the maximum likelihood (ML) approach. Therefore, the resulting receiver is optimum and does not suffer from the difficulties resulted from direct application of the ML procedure. One of these difficulties is the computational complexity which depends exponentially on the data sequence length. Introducing an iterative structure in the developed MRC achieves a linear computational complexity and enables efficient data extraction by the Viterbi algorithm when trellis coding is used.
\end{abstract}

Keywords: Diversity, Expectation-maximization algorithm, Maximal ratio combiner.

\section{INTRODUCTION}

It is well known that diversity is an efficient method for combating fading effects [1]. Several combining techniques have been proposed to illustrate the improvement in signal statistics $[2,3]$. The main diversity combining techniques are selection diversity, equal gain combining and MRC. Selection diversity is the simplest of these techniques $[4,5]$. In this technique, the receiver monitors the signal-to-noise ratio of all branches and selects and uses the information from the branch with the largest SNR. Equal gain combining requires the receiver to coherently sum the signals received through all channels in order to increase the available signal-to-noise ratio at the receiver [6]. MRC is an optimum spatial diversity strategy to reduce the signal fluctuation caused by fading [7]. Maximal ratio combining always perform better than either selection diversity or equal gain combining because it is an optimum combiner. The information on all channels is used with this technique to get a more reliable received signal. This method is known to be theoretically optimal for slow fading in the sense that it gives the best statistical reduction of fading in any linear diversity combiner [8-10]. In [11], it is proved that an MRC operating on correlated branches is optimal even if the branch signals are weighted as though they are independent. However, the fast fading channel introduces additional pulse distortion which must be removed to avoid inter-symbol interference [12]. This problem is solved in [13] and the performance of the MRC in this case is improved. The performance of the MRC is affected by several factors; one of them is the error in determining the gain factor of the diversity branches of the MRC. In [14], some results regarding the effects of the gain factor estimation error on the performance of MRC are reported. Also, it

\footnotetext{
*Address correspondence to this author at the Electrical Engineering Department, MTC, Cairo, 821 Zahraa Nasr City, Flat 31, Nasr City, Cairo, Egypt; Tel: +2 02 24098320; E-mail: elmahdy77@ hotmail.com
}

is reported in [14] that a pilot signal, transmitted with the data, can be used to provide an estimate of the combiner gain factors.

In this paper, the EM algorithm is used to estimate the gain factors of the combiner without using pilot signal transmission. Also, the EM algorithm is used to recover the transmitted sequence. The signals are assumed to have constant envelope (like MPSK), transmitted over a Rayleigh fading channel and contaminated with additive white Gaussian noise (AWGN). The developed MRC has an iterative form which reduces the complexity of the ML diversity receiver that arises from the difficulty of performing the maximization of the likelihood function. This difficulty becomes significant when $M$-ary signaling with long sequences is transmitted. In this case, exhaustive search is used to obtain this maximization. For example, for a sequence of length $K$ transmitted on $L$ channels with $M$-ary signaling, the required number of operations resulted from direct applications of ML approach is $L M^{K}$. This number is reduced to $L M$ using the developed MRC.

The rest of the paper is organized as follows. Section 2 provides the derivation of the developed MRC. In section 3, simulations are presented to demonstrate the performance of the MRC. Finally, conclusions are presented in section 4.

\section{MRC DERIVATION}

In this section, the gain factors of the MRC in addition to the estimated sequence are estimated using the EM algorithm. The transmitted signal is received over $L$ statistically independent and identically distributed ( i.i.d) fading channels, each of them being a slowly varying flat fading as shown in Fig. (1). In this figure, $z_{l}(t) ; l=1,2, \ldots, L$, is the set of received replicas of a signal, where $l$ is the channel index and $D_{l}$ is the $l$-th channel fading parameter. The fading pa- 


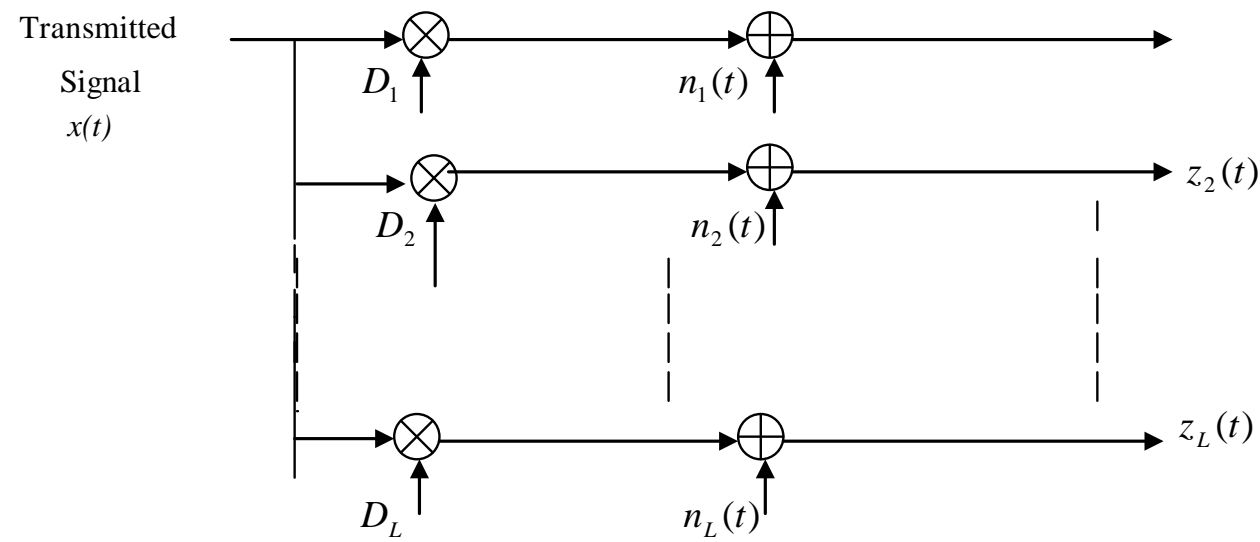

Fig. (1). Channel model.

rameter $D_{l}=\alpha_{l} e^{j \phi_{l}}$ is a zero mean complex Gaussian random variable with a Rayleigh distributed amplitude $\alpha_{l}$ and uniform distributed phase angle $\phi_{l}$. The variance of the random variable $D_{l}$ is assumed to be $\sigma_{l}^{2}$. Furthermore, the fading signals are perturbed by a zero mean complex AWGN with variance $N_{o} / 2$. The noise is assumed to be statistically independent from channel to channel and independent of the fading amplitudes. Moreover, it is assumed that the noise has equal power at each branch. For each diversity channel, the signal at the receiver input has the form:

$z_{l}(t)=D_{l} x(t)+n_{l}(t)$

where $n_{l}(t)$ is a zero mean additive white Gaussian noise process and $x(t)$ is a constant envelope signal. After matched filtering and sampling, the sampled received signal $z_{l, k}(k)$ can be written as

$z_{l, k}=D_{l} x_{k}+n_{l, k}$

where $n_{l, k}$ is a sample of zero-mean complex Gaussian noise, and $x_{k}$ is the $k^{\text {th }}$ symbol of the received signal. After suitable normalization and without loss of generality, it is assumed that for all $k, E\left(x_{k}\right)=0, E\left(\left|x_{k}\right|^{2}\right)=1$, and that the variance of $n_{l, k}$ is $N_{o} / 2$. Let a length $K$ of the signal symbol sequence $x_{1}, x_{2}, \ldots, x_{K}$, be represented by the vector $\mathbf{x}$, then the received vector of the $l^{\text {th }}$ iteration channel, $\mathbf{z}_{l}=\left(z_{l, 1}, z_{l, 2}, \ldots, z_{l, K}\right)^{T}$, can be expressed as:

$\mathbf{z}_{l}=D_{l} \mathbf{x}+\mathbf{n}_{l}$

where $\mathbf{n}_{l}=\left(n_{l, 1}, n_{l, 2}, \ldots, n_{l, K}\right)^{T}$ is a zero-mean i.i.d, complex, Gaussian noise vector. The likelihood function (LF) of the data $\mathbf{z}_{l}$, normalized to the probability density function of the noise, is given by:

$$
f\left(\mathbf{z}_{l} \mid D_{l}, \mathbf{x}\right)=\frac{\frac{1}{\left(\pi N_{o}\right)^{K}} \exp \left\{\frac{-\left\|\mathbf{z}_{l}-D_{l} \mathbf{x}\right\|^{2}}{N_{o}}\right\}}{\frac{1}{\left(\pi N_{o}\right)^{K}} \exp \left\{\frac{-\left\|\mathbf{z}_{l}\right\|^{2}}{N_{o}}\right\}}
$$

Direct application of the ML procedure is to maximize $f(\mathbf{Z} \mid \mathbf{D}, \mathbf{x})=\prod_{l=1}^{L} f\left(\mathbf{z}_{l} \mid D_{l}, \mathbf{x}\right)$ with respect to the fading vector $\mathbf{D}$ and the data vector $\mathbf{x}$, where $\mathbf{D}=\left(D_{1}, D_{2}, \ldots, D_{L}\right)^{T}$ and $\mathbf{Z}=\left(\mathbf{z}_{1}, \mathbf{z}_{2}, \ldots, \mathbf{z}_{L}\right)^{T}$. This maximization can not be computed in closed form. However, one of the available solutions to this problem is to obtain $\hat{\mathbf{D}}$ for a given data sequence and then evaluate $f(\mathbf{Z} \mid \mathbf{D}, \mathbf{x})$ for all possible data sequences and choose the data sequence that maximizes it. For $M$-ary signaling, the required number of operations to perform this search is $L M^{K}$. This exhaustive search is time consuming and complicated. Therefore, it is required to develop another receiver that has a smaller complexity than this receiver. We propose the MRC receiver shown in Fig. (2), in which the combiner gains and the data are estimated using the EM algorithm. This receiver achieves a linear computational complexity. In ideal practical MRC, each matched filter output is multiplied by the corresponding combiner gain factor. The effect of this multiplication is to compensate for the phase shift in the channel and to weight the signal by a factor that is proportional to the signal strength. Thus a strong signal carries a larger weight than a weak signal. In the following, we will use the EM algorithm to derive this receiver.

Using the relation $\left\|\mathbf{z}_{l}-D_{l} \mathbf{x}\right\|^{2}=\sum_{k=1}^{K}\left|z_{l, k}-D_{l} x_{k}\right|^{2}$, the likelihood function (LF) of the data $\mathbf{z}_{l}$, can be simplified as

$$
f\left(\mathbf{z}_{l} \mid D_{l}, \mathbf{x}\right)=\exp \left\{\frac{2}{N_{o}} \sum_{k=1}^{K} \operatorname{Re}\left(z_{l, k}^{*} \quad x_{k} D_{l}\right)-\frac{1}{N_{o}}\left|D_{l}\right|^{2} \sum_{k=1}^{K}\left|x_{k}\right|^{2}\right\}
$$




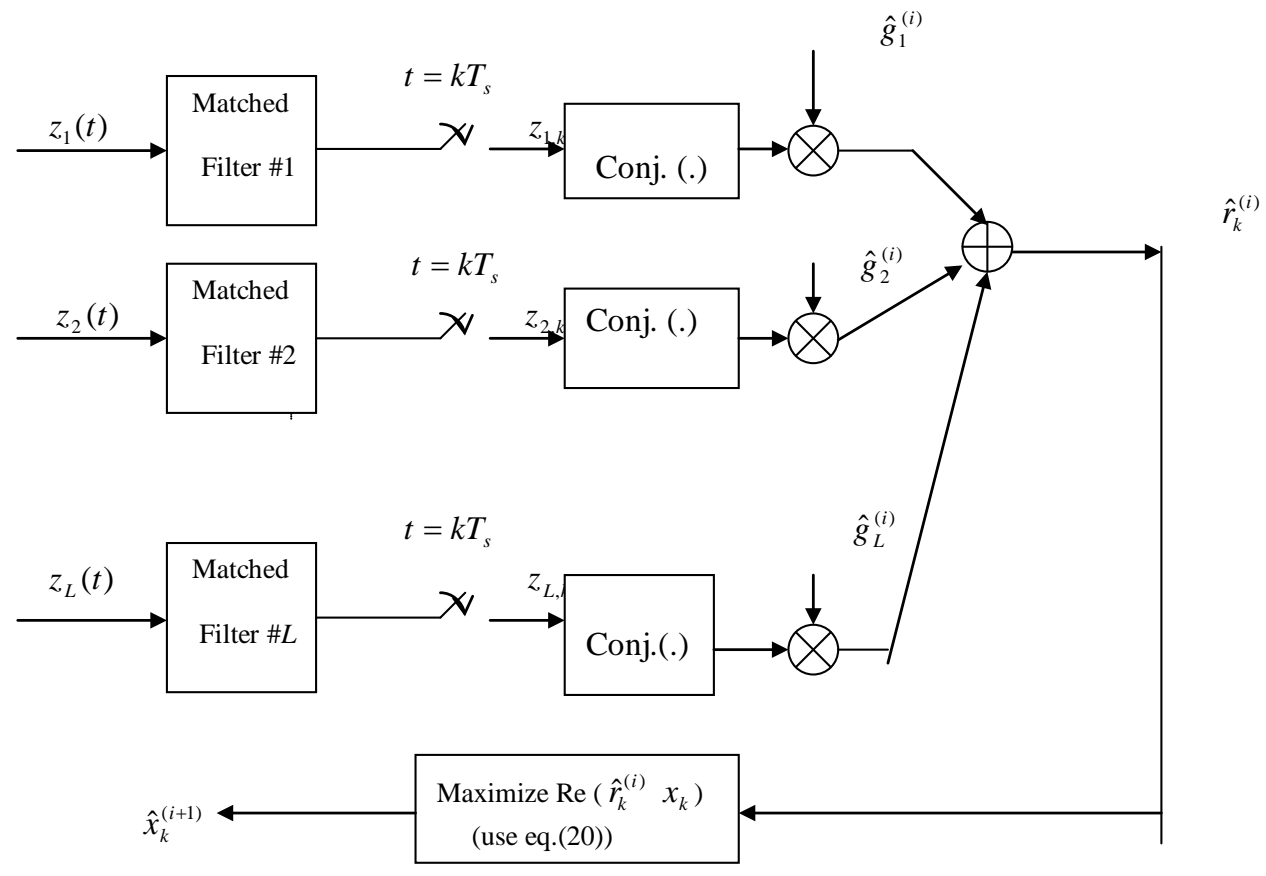

Fig. (2). The developed maximal ratio combiner.

where $*$ denotes the complex conjugate. For constant envelope signals the second term in (5) does not affect the maximization and can be dropped. In our problem the combiner gains are given by $\hat{g}_{l}=\hat{D}_{l} ; l=1,2, \ldots, L$, where $\hat{D}_{l}$ is the estimated channel parameter at the $l^{\text {th }}$ branch. To use the EM algorithm, we need to specify the incomplete and the complete data. In our problem, the incomplete data is the $L \times K$ observation matrix $\mathbf{Z}$ and the complete data is chosen to be $\mathbf{V}=(\mathbf{Z}, \mathbf{D})$. Since $\mathbf{x}$ and $\mathbf{D}$ are independent, the conditional density function of the complete data $\mathbf{V}$ given $\mathbf{x}$ is given by:

$$
f(\mathbf{V} \mid \mathbf{x})=f(\mathbf{Z}, \mathbf{D} \mid \mathbf{x})=f(\mathbf{Z} \mid \mathbf{D}, \mathbf{x}) f(\mathbf{D} \mid \mathbf{x})
$$

Then, at the $i^{\text {th }}$ iteration, the E-step of the EM algorithm can be written as:

$U^{\prime}\left(\mathbf{x} \mid \hat{\mathbf{x}}^{(i)}\right)=E\left(\log f(\mathbf{Z} \mid \mathbf{D}, \mathbf{x}) \mid \mathbf{Z}, \hat{\mathbf{x}}^{(i)}\right)+E\left(\log f(\mathbf{D} \mid \mathbf{x}) \mid \mathbf{Z}, \hat{\mathbf{x}}^{(i)}\right)$

where $\hat{\mathbf{x}}^{(i)}$ is the most recent sequence estimate at the $i$-th iteration of the EM algorithm. The conditional expectation in (6) is with respect to the conditional density of the complex combiner gain factors vector $\mathbf{D}$ given the incomplete data and assuming that $\mathbf{x}=\hat{\mathbf{x}}^{(i)}$. The second term in (7) is constant because it is not a function of $\mathbf{x}$ and can be dropped without affecting the maximization step. The conditional density $f(\mathbf{Z} \mid \mathbf{D}, \mathbf{x})$ is given by:

$$
f(\mathbf{Z} \mid \mathbf{D}, \mathbf{x})=\prod_{l=1}^{L} f\left(\mathbf{z}_{l} \mid D_{l}, \mathbf{x}\right)=\prod_{l=1}^{L} \exp \left\{\frac{2}{N_{o}} \sum_{k=1}^{K} \operatorname{Re}\left(z_{l, k}^{*} x_{k} D_{l}\right)\right\}
$$

Using (7), the E-step of the EM algorithm at the $i^{\text {th }}$ iteration can be written as:

$U\left(\mathbf{x} \mid \hat{\mathbf{x}}^{(i)}\right)=\sum_{l=1}^{L} E\left\{\sum_{k=1}^{K} \operatorname{Re}\left(z_{l, k}^{*} x_{k} D_{l}\right) \mid \mathbf{Z}, \hat{\mathbf{x}}^{(i)}\right\}$

The conditional expectation in the E-step is with respect to the conditional density of the complex fading parameter $D_{l}$ given the incomplete data $\mathbf{z}_{l}$ and assuming that $\mathbf{x}=\hat{\mathbf{x}}^{(i)}$. This E-step can be written as:

$U\left(\mathbf{x} \mid \hat{\mathbf{x}}^{(i)}\right)=\sum_{l=1}^{L}\left(\sum_{k=1}^{K}\left(\operatorname{Re}\left(z_{l, k}^{*} x_{k} \hat{D}_{l}^{(i)}\right)\right)\right)$

where $\hat{D}_{l}^{(i)}=E\left(D_{l} \mid \mathbf{z}_{l}, \hat{\mathbf{x}}^{(i)}\right)$ is the conditional mean of $D_{l}$ given the incomplete data $\mathbf{z}_{l}$ and assuming that $\mathbf{x}=\hat{\mathbf{x}}^{(i)}$ and it is independent of $\mathbf{x}$. In the following, the expressions of $\hat{D}_{l}^{(i)}$ will be derived and requires obtaining the conditional density $f\left(D_{l} \mid \mathbf{z}_{l}, \hat{\mathbf{x}}^{(i)}\right)$ which can be expressed as:

$f\left(D_{l} \mid \mathbf{z}_{l}, \hat{\mathbf{x}}^{(i)}\right)=f\left(\mathbf{z}_{l} \mid D_{l}, \hat{\mathbf{x}}^{(i)}\right) f\left(D_{l}\right)$

Using (8), the conditional density $f\left(\mathbf{z}_{l} \mid D_{l}, \hat{\mathbf{x}}^{(i)}\right)$ can be written as:

$f\left(\mathbf{z}_{l} \mid D_{l}, \hat{\mathbf{x}}^{(i)}\right)=\exp \left\{\frac{2}{N_{o}} \sum_{k=1}^{K} \operatorname{Re}\left(z_{l, k}^{*} \hat{x}_{k}^{(i)} D_{l}\right)\right\}$

Knowing that $D_{l}$ is a zero mean complex Gaussian random variable with variance $\sigma_{l}^{2}$ and by substitution of 
$f\left(\mathbf{z}_{l} / D_{l}, \hat{\mathbf{x}}^{(i)}\right)$ in (11), the expression of $f\left(D_{l} \mid \mathbf{z}_{l}, \hat{\mathbf{x}}^{(i)}\right)$ can be written as:

$f\left(D_{l} \mid \mathbf{z}_{l}, \hat{\mathbf{x}}^{(i)}\right)=\frac{1}{2 \pi \sigma_{l}^{2}} \exp \left\{\frac{2}{N_{o}} \sum_{k=1}^{K} \operatorname{Re}\left(z_{l, k}^{*} \hat{x}_{k}^{(i)} D_{l}\right)\right\} \exp \left\{\frac{-1}{2 \sigma_{l}^{2}}\left|D_{l}\right|^{2}\right\}$

which, after some algebraic manipulations, can be written as:

$$
f\left(D_{l} \mid \mathbf{z}_{l}, \hat{\mathbf{x}}^{(i)}\right)=c \exp \left\{\frac{-1}{2 \sigma_{l}^{2}}\left|D_{l}-\frac{2 \sigma_{l}^{2}}{N_{o}} \sum_{k=1}^{K} z_{l, k} \hat{x}_{k}^{*(i)}\right|^{2}\right\}
$$

where $\mathrm{c}$ is a constant which needs not to be calculated. Then from (14), the gain factor of the $l^{\text {th }}$ branch of the MRC is given by:

$\hat{g}_{l}^{(i)}=\hat{D}_{l}^{(i)}=E\left\{D_{l} \mid \mathbf{z}_{l}, \hat{\mathbf{x}}^{(i)}\right\}=2 \gamma_{c} \sum_{k=1}^{K} z_{l, k} \hat{x}_{k}^{*(i)}$

where $\gamma_{c}=\frac{\sigma_{l}^{2}}{N_{o}}$ is the average signal to noise ratio per channel. It is clear that the estimate of the combiner gain factors is optimum since, $\hat{D}_{l}^{(i)}$ is obtained by maximizing the LF given by (14). Also, from (15) it is noted that, the estimation of the combiner gain factors is determined without sending a pilot signal with the data. They are function of the received signal, the estimated symbol sequence, and $\gamma_{c}$. Unfortunately, $\gamma_{c}$ is not known in practice and needs to be estimated at the receiver. There are several methods to estimate $\gamma_{c}$. A blind (does not require a training sequence) and online method for fading channel with $L$ branches is described in [15]. In this method, $\gamma_{c}$ is derived using statistical ratio of certain observables over a block of data. The method is derived for a Nakagami- $m$ channel and can be used in a Rayleigh fading channel as special case by using $m=1$.

Now, we obtain the symbol sequence at the $i^{\text {th }}$ iteration which is derived by applying the E-step of the EM algorithm. Using (10) and (15), the E-step at the $i^{\text {th }}$ iteration can be written as:

$U\left(\mathbf{x} \mid \hat{\mathbf{x}}^{(i)}\right)=\sum_{k=1}^{K}\left(\sum_{l=1}^{L}\left(\operatorname{Re}\left(z_{l, k}^{*} x_{k} \hat{g}_{l}^{(i)}\right)\right)\right)$

In this case, the inner summation in (16) represents the combined diversity channel output at the $i^{\text {th }}$ iteration multiplied by $x_{k}$ as shown in Fig. (2). That is, the combined diversity channel output at the $i^{\text {th }}$ iteration can be viewed as $\hat{r}_{k}^{(i)}=\sum_{l=1}^{L} z_{l, k}^{*} \hat{g}_{l}^{(i)}$ which is equivalent to MRC [1]. Now the maximization step of the EM algorithm is carried out by maximization of $U\left(\mathbf{x} / \hat{\mathbf{x}}^{(i)}\right)=\sum_{k=1}^{K}\left(\operatorname{Re}\left(\hat{r}_{k}^{(i)} x_{k}\right)\right)$. Note that maximizing $U\left(\mathbf{x}, \hat{\mathbf{x}}^{(i)}\right)$ with respect to the sequence $\mathbf{x}$ is equivalent to maximizing each symbol in the sum i.e. mak- ing symbol by symbol decision. Then, the M-step of the EM algorithm is given by: Compute for $k=1,2, \ldots, K$

$$
\hat{x}_{k}^{(i+1)}=\arg \max _{x_{k}} \sum_{l=1}^{L} \operatorname{Re}\left(z_{l, k}^{*} x_{k} \hat{g}_{l}^{(i)}\right)
$$

which can be efficiently performed using Viterbi algorithm when trellis coding is used. The algorithm starts at $i=0$ and assumes the initial value for the gain factors $\hat{g}_{l}^{(0)}, l=1,2, \ldots, L$. Then, we obtain $\hat{x}_{k}^{(i+1)}$ using (17) and we use its value in (15) to obtain the gain factors at the next iteration. These steps are repeated until the algorithm converges.

\section{COMPUTER SIMULATIONS AND RESULTS}

In this section, the theoretical developments presented above are validated by simulation experiments. The accuracy of the estimation algorithm of the MRC gain factors is demonstrated. Moreover, the performance of the developed MRC is evaluated and is compared with the MRC which uses pilot symbols for channel estimation. The performance is measured in terms of the bit error probability as a function of the average signal to noise ratio per channel $\left(\gamma_{c}\right)$. The signal used in the simulations is BPSK. The signal is transmitted on a Rayleigh flat fading channels using independent complex Gaussian random generators. The carrier frequency of the signals is $0.5 \mathrm{MHz}$ and the sampling frequency is $5 \mathrm{MHz}$. The number of fading channels is varied from 1 to 7 . The number of samples is 4096 . The signals are added to a generated white Gaussian noise with variance $N_{0} / 2$.

\subsection{Performance Evaluation of the Estimation Algorithm}

First, the accuracy of the estimation algorithm is demonstrated in Figs. (3, 4, and 5). Figs. (3 and $\mathbf{4})$ are plotted at the third iteration of the algorithm. The true and estimated values of three MRC gain factors $\left|\hat{g}_{1}\right|,\left|\hat{g}_{2}\right|$, and $\left|\hat{g}_{3}\right|$ are shown in Fig. (3). In this figure, the estimated values correspond to the dashed lines while the true values correspond to the solid lines. This figure shows that as $\gamma_{c}$ increases, the values of the estimated gain factors $\left|\hat{g}_{1}\right|,\left|\hat{g}_{2}\right|$, and $\left|\hat{g}_{3}\right|$ converge to their true values. The plot of the normalized mean square error (NMSE) of estimation of $\left|\hat{g}_{1}\right|,\left|\hat{g}_{2}\right|$ and $\left|\hat{g}_{3}\right|$ is shown in Fig. (4). The results show that, at low SNR, the NMSE is high because the noise dominates the performance of the estimator and as SNR increases, the NMSE decreases. The convergence of the algorithm for $\mathrm{SNR}=-1$ and $5 \mathrm{~dB}$ is illustrated in Fig. (5). In this figure, the true and estimated values of $\left|\hat{g}_{1}\right|,\left|\hat{g}_{2}\right|$ and $\left|\hat{g}_{3}\right|$ are plotted versus the number of iterations. This figure shows that the estimation algorithm converges to the true value within two or three iterations. This figure also shows that at $\mathrm{SNR}=5 \mathrm{~dB}$, the convergence to the true values is faster than the convergence at $\mathrm{SNR}=-1 \mathrm{~dB}$. For example, at $\mathrm{SNR}=5 \mathrm{~dB}$, the estimated curve for $\left|\hat{g}_{2}\right|$ 
converges within three iterations while at $\mathrm{SNR}=5 \mathrm{~dB}$, it converges within two iterations.

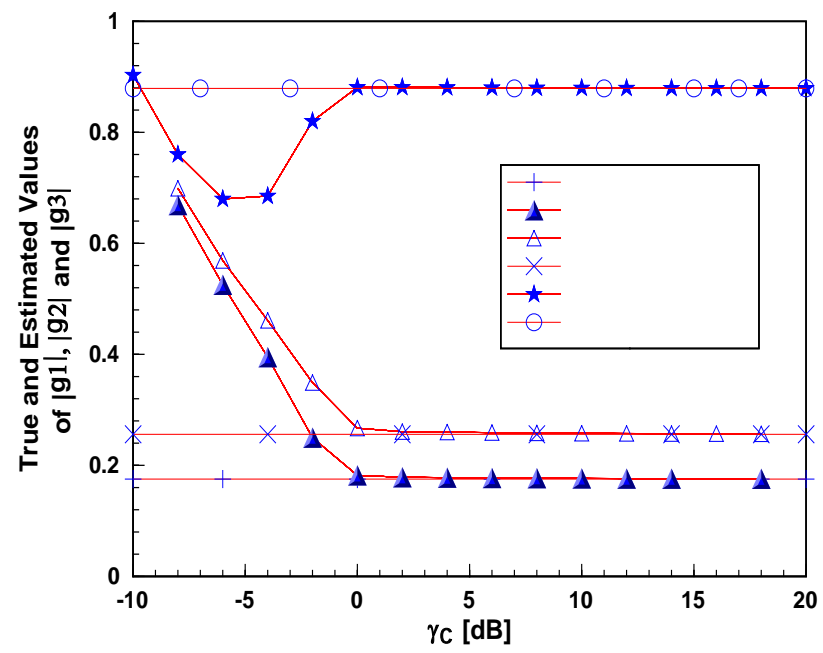

Fig. (3). True and estimated values of the absolute of the combiner gain factors $\left|g_{1}\right|,\left|g_{2}\right|$, and $\left|g_{3}\right|$ versus the average signal to noise ratio per channel.

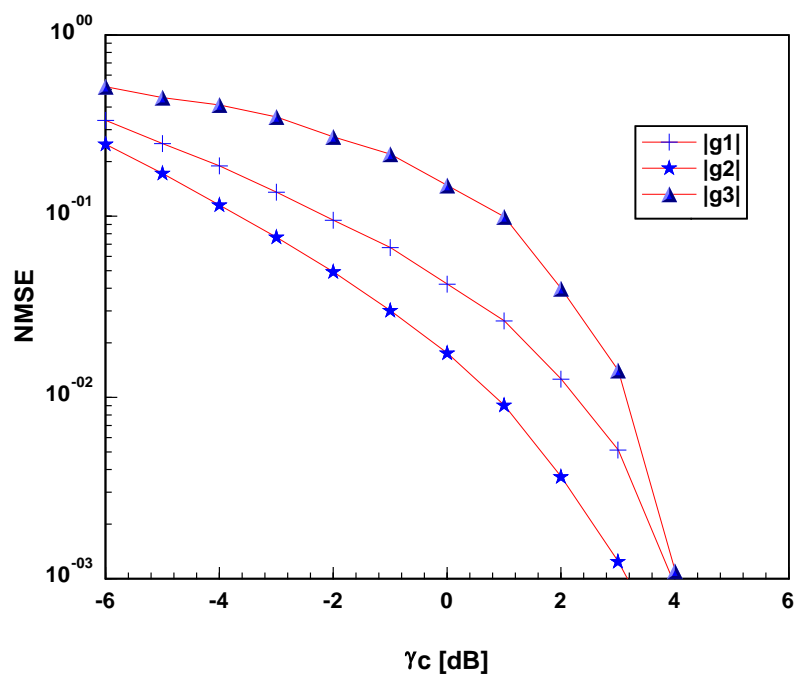

Fig. (4). Normalized mean error estimation of the absolute of the combiner gain factors $\left|g_{1}\right|,\left|g_{2}\right|$, and $\left|g_{3}\right|$.

\subsection{Performance Evaluation of the Developed MRC}

Now, the performance of the developed MRC is investigated. The bit error probability versus the signal to noise ratio per channel is shown in Fig. (6) for independent diversity with $L=1,3,4$, and 7 branches. This figure is also plotted at the third iteration of the algorithm. The figure shows that as the average signal to noise ratio per channel increases, the bit error probability decreases. At low $\gamma_{c}$, the noise dominates the performance of the MRC and the bit error probability becomes high. When $\gamma_{c}$ increases, the effectiveness of diversity becomes significant, that is, higher diversity (as $L$ increases) can significantly reduce the bit error probability which illustrates the advantage of the diversity. For example, at $L=1$, the MRC requires $12 \mathrm{~dB}$ SNR to obtain a bit error probability $10^{-4}$ but this value of bit error probability can be reached at $\mathrm{SNR}=1 \mathrm{~dB}$ for $L=7$.

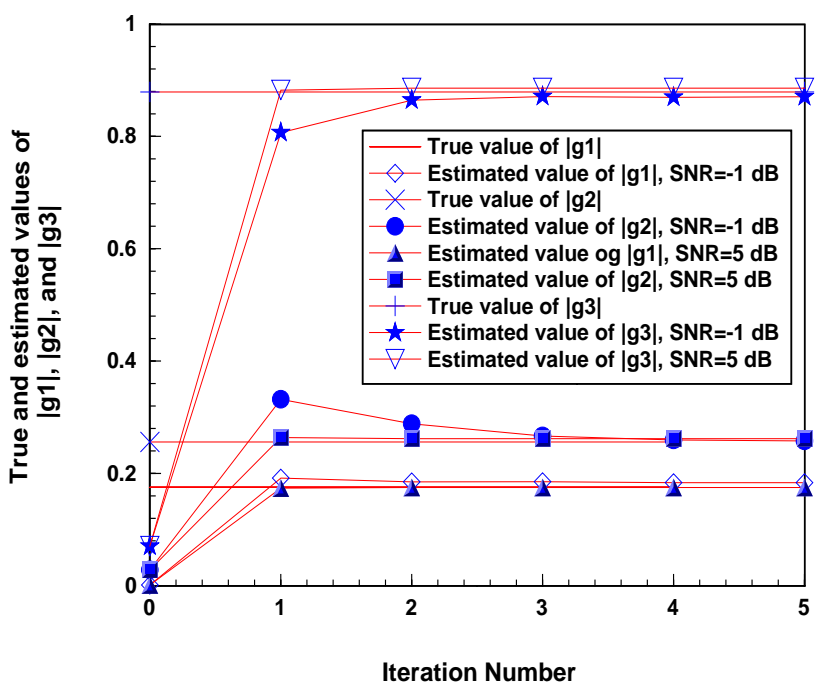

Fig. (5). True and estimated values of the absolute of the combiner gain factors $\left|g_{1}\right|,\left|g_{2}\right|$, and $\left|g_{3}\right|$ versus the number of iterations.

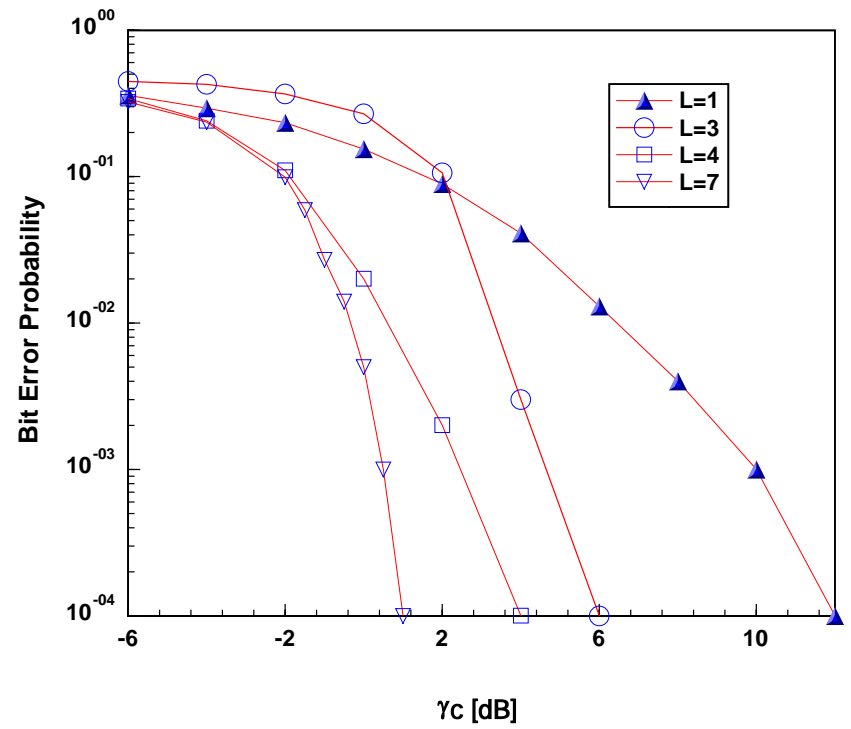

Fig. (6). Performance of the proposed receiver.

The performance comparison between the proposed MRC and the MRC which uses pilot symbols to estimate the channel is evaluated in terms of bit error probability versus $\gamma_{c}$. In MRC with pilot aided channel estimation, the ML algorithm is used to recover the transmitted data; therefore we call it MRC/ML. The MRC/ML has optimum performance and is considered as a reference receiver. In MRC/ML, the least square algorithm is used to estimate the fading 
channel parameters. The number of the used pilot symbols is 50 which is found to obtain a reliable estimate. The outputs of the matched filters are multiplied by the complex conjugate of the resulting channel parameters estimation. The real part of the combined weighted matched filters outputs is maximized to decide the transmitted sequence. The results of the comparison are shown in Fig. (7). This figure shows that there is a gap in performance between the MRC/ML and the proposed MRC. The MRC/ML offers a performance gain over the proposed MRC. For $L=7$ and a bit error probability $10^{-4}$, the value of this gap is around $0.5 \mathrm{~dB}$. The reason for this gap is that there is a loss in performance in the proposed MRC due to the iterations of the EM algorithm.

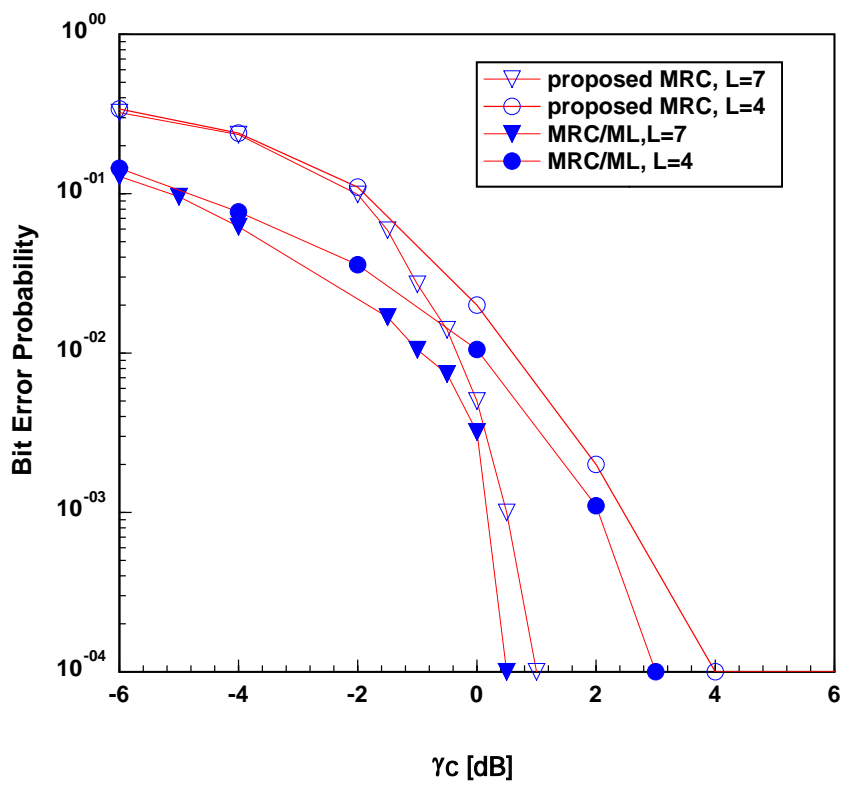

Fig. (7). Performance comparison of the proposed MRC and the $\mathrm{MRC} / \mathrm{ML}$.

\section{CONCLUSIONS}

EM-based MRC has been derived for noisy constant envelope signals. The signals are received through flat fading multi-channel. The EM algorithm estimates the gain factors of the MRC and also the transmitted sequence. Due to its iterative nature, the derived $\mathrm{MRC}$ has lower complexity than the ML diversity receiver. It MRC has a linear computational complexity rather than the exponential complexity achieved by the ML diversity receiver. Although, the developed MRC has an iterative nature, it converges within two to three iterations but it has a performance loss about $0.5 \mathrm{~dB}$ from the MRC with pilot aided channel estimation.

\section{REFERENCES}

[1] Y. Ma, Q. Zhang, R. Schober, and S. Pasupathy, "Diversity reception of DAPSK over generalized fading channels", IEEE Trans. Wireless Commun., vol. 4, pp. 1834-1846, July 2005.

[2] T. Eng, N.Kong, and L.B.Milstein, "Comparison of diversity combining techniques for Rayleigh-fading channels", IEEE Trans. Commun., vol. 44, pp. 1117-1129, Sept. 1996.

[3] W. Jingxian, and X. Chengshan, "Optimal diversity combining based on linear estimation of rician fading channels", IEEE Trans. Commun., vol. 56, pp. 1612-1615, Oct. 2008.

[4] N. Kong, and L. Milstein, "SNR of generalized diversity selection combining with nonidentical Rayleigh fading statistics", IEEE Trans. Commun, vol. 48, pp. 1266-1271, Aug. 2000.

[5] N. Beaulieu, "Switching rates of dual selection diversity and dual switch-and-stay diversity", IEEE Trans. Commun., vol. 56, pp. 1409-1413, Sept. 2008.

[6] D. Zogas, G. Karagiannidis, and S. Kotsopoulos, "Equal gain combining over Nakagami-n (rice) and Nakagami-q (Hoyt) generalized fading channels", IEEE Trans. Wireless Commun., vol. 4, pp. 374 379, March 2005.

[7] T. Lin, "Maximal ratio combining for iterative multiuser decoding", M. S. thesis, University of south Australia, Australia, 2005.

[8] J. Proakis, Digital Communications, New York: McGraw-Hill, 2000.

[9] A. Annamalai, "Micro diversity reception of spread spectrum signals on Nakagami fading channels", IEEE Trans. Commun., vol. 47, pp.1747-1756, Nov. 1999.

[10] H. Yang, and M. Alouini, "MRC and GSC diversity combining with an output threshold", IEEE Trans. Veh. Technol., vol. 54, pp. 1081-1090, May 2005.

[11] X. Dong and N. Beaulieu, "Optimal maximal ratio combining with correlated diversity branches", IEEE Commun. Lett., vol. 1, pp.2224, Jan. 2002.

[12] A. El-Mahdy, "Adaptive Channel Estimation and Equalization for Rapidly Mobile Communication Channels", IEEE Trans. Commun, vol. 52, pp. 1162-1135, July 2004

[13] B. Hart, and D. Taylor, "Extended MLSE diversity receiver for time and frequency selective channel", IEEE Trans. Commun., vol. 45, pp.322-333, Mar. 1997.

[14] B. Tomiuk, N. Beaulieu, and A. Dayya, "General forms for maximal ratio diversity with weighting errors", IEEE Trans. Commun., vol. 47, pp.488- 492, Apr. 1999.

[15] A. Ramesh, A. Chockalingam, and L. Milstein, "SNR estimation in Nakagami- $m$ fading with diversity combining and its application to turbo decoding", IEEE Trans. Commun., vol. 50, pp. 1719- 1724, Nov. 2002.

(C) Ahmed El-Sayed El-Mahdy; Licensee Bentham Open.

This is an open access article licensed under the terms of the Creative Commons Attribution Non-Commercial License (http://creativecommons.org/licenses/by-nc/3.0/) which permits unrestricted, non-commercial use, distribution and reproduction in any medium, provided the work is properly cited. 